\title{
Die Wahlen zum dänischen Folketing vom 18. Juni 2015: knapper bürgerlicher Sieg bei erdrutschartigen Wählerwanderungen
}

Article

Accepted Version

Arndt, C. (2016) Die Wahlen zum dänischen Folketing vom 18. Juni 2015: knapper bürgerlicher Sieg bei erdrutschartigen Wählerwanderungen. Zeitschrift für Parlamentsfragen, 47 (4). pp. 771-782. ISSN 0340-1758 doi:

https://doi.org/10.5771/0340-1758-2016-4-771 Available at https://centaur.reading.ac.uk/73135/

It is advisable to refer to the publisher's version if you intend to cite from the work. See Guidance on citing.

Published version at: https://www.nomos-elibrary.de/10.5771/0340-1758-2016-4-771/die-wahlen-zum-daenischenfolketing-vom-18-juni-2015-knapper-buergerlicher-sieg-bei-erdrutschartigen-waehlerwanderungen-jahrgang-47-2016heft-4

To link to this article DOI: http://dx.doi.org/10.5771/0340-1758-2016-4-771

Publisher: Nomos Verlagsgesellschaft

All outputs in CentAUR are protected by Intellectual Property Rights law, including copyright law. Copyright and IPR is retained by the creators or other copyright holders. Terms and conditions for use of this material are defined in the End User Agreement. 


\section{CentAUR}

Central Archive at the University of Reading

Reading's research outputs online 
Die Wahlen zum dänischen Folketing vom 18. Juni 2015: knapper bürgerlicher Sieg bei erdrutschartigen Wählerwanderungen

Manuskript für Heft 4/2016 in der Zeitschrift für Parlamentsfragen

31. Oktober 2016

\author{
Christoph Arndt, $\mathrm{PhD}$ \\ arndt@ps.au.dk \\ Institut for Statskundskab \\ Aarhus Universitet \\ Bartholins Allé 7 \\ 8000 Aarhus C \\ Dänemark
}

Anzahl der Zeichen: 36.111 


\section{Die Wahlen zum dänischen Folketing vom 18. Juni 2015: knapper bürgerlicher Sieg bei erdrutschartigen Wählerwanderungen}

\section{Christoph Arndt}

Seit der Folketingswahl 2011 gab es nach zehnjähriger bürgerlicher Dominanz wieder eine mittelinks Minderheitsregierung, die von den Sozialdemokraten angeführt wurde. ${ }^{1}$ Diese Regierung, die zunächst aus einer Koalition aus Sozialdemokraten, Sozialliberalen und der Sozialistischen Volkspartei bestand, die von der linksradikalen Einheitsliste toleriert wurde, wurde jedoch bei der Wahl im Juni 2015 nicht im Amt bestätigt. Dies war auch die Konsequenz aus zahlreichen internen Auseinandersetzungen, Kabinettsumbildungen und dem Bruch zentraler Wahlversprechen auf Seiten der Sozialdemokraten und Sozialisten, welche im Austritt der Sozialisten aus der Regierungskoalition Anfang 2014 kulminierten. Im Gegensatz zu ihrer liberal-konservativen Vorgängerregierung war die sozialdemokratische Minderheitsregierung häufig auf blocküberschreitende Zusammenarbeit mit den Oppositionsparteien angewiesen, da sich die Einheitsliste den zahlreichen Sozialstaatsreformen in der Legislaturperiode 2011-2015 verweigerte, welche auch ein Grund für die gescheiterte Wiederwahl der sozialdemokratisch-sozialliberalen Minderheitsregierung darstellen. Die Wahl 2015 war durch die größte Nettovolatilität seit 1973 geprägt und brachte die nationalkonservative Dänische Volkspartei als großen Sieger hervor, während die rechtsliberale Venstre ihre Position als stärkste Partei an die Sozialdemokraten abgeben musste und nur noch die drittstärkte Partei ist. Die Dänische Volkspartei wurde zum ersten Mal die stärkste bürgerliche Partei, somit konnte der europaweite Trend zu einer Stärkung nationalkonservativer Parteien auch bei der Folketingswahl 2015 beobachtet werden. Die nach der Wahl 2015 gebildete liberale Minderheitsregierung wird zum ersten Mal seit 1973 von der drittstärksten Partei alleine gebildet. Ihr langfristiges Überleben wird von ihrer Manövrierfähigkeit und der Fähigkeit, Kompromisse mit den anderen bürgerlichen Parteien einzugehen, abhängen. Darüber hinaus wurden die Ausnahmeregelungen für die sogenannten Rechtsvorbehalte Dänemarks in der Europäischen Union in einem Referendum am 3. Dezember 2015 bestätigt, was als Niederlage für die liberale Minderheitsregierung gewertet werden kann.

\footnotetext{
${ }^{1}$ Zur Wahl zum Folketing 2011 Christoph Arndt, Die Folketingswahl in Dänemark vom 15. September 2011: Abwahl einer Regierung ohne klaren Sieg der Opposition, in: Zeitschrift für Parlamentsfragen, 43. Jg. (2012), H. 1, S. 142 152.
} 


\section{Die Ausgangslage}

Seit der Wahl zum Folketing vom 15. September 2011 wurde Dänemark von einer sozialdemokratisch-sozialliberalen Minderheitsregierung regiert, der bis zum 30 Januar 2014 auch die Sozialistische Volkspartei (SF) angehörte. Diese Regierung wurde von der linksradikalen Einheitsliste (Enhedslisten, EL) und nach ihrem Ausscheiden aus der Regierung auch von der SF toleriert. Die mitte-links Regierung hatte somit eine faktische, wenn auch äußerst knappe parlamentarische Grundlage und schöpfte fast die maximal zulässige Dauer einer Legislaturperiode von vier Jahren aus. Hier ist anzumerken, dass die Wahlperiode für eine dänische Regierung maximal vier Jahre beträgt, der Ministerpräsident im Gegensatz zum deutschen Kanzler aber vorzeitige Neuwahlen ohne Misstrauensvotum oder eine Vertrauensfrage ausschreiben kann. Darüber hinaus sind Minderheitenregierungen in Dänemark die Norm, da lediglich vier Kabinette seit 1953 auch eine eigene parlamentarische Mehrheit nach der Folketingswahl ohne Tolerierung durch andere Parteien hatten (die Kabinette Hansen II, Kampmann II, Baunsgaard und Nyrup Rasmussen I). ${ }^{2}$ Die am 27. Mai ausgerufenen Wahlen zum Folketing am 18. Juni bedeuteten dann das Ende einer von Beginn an turbulenten Regierungsperiode.

Die von Ministerpräsidentin Helle Thorning-Schmidt geführte Regierung aus Sozialdemokraten (S), Sozialliberalen (det Radikale Venstre, RV) und der Sozialistische Volkspartei (SF) hatte bereits kurz nach der Regierungsübernahme mit einem Glaubwürdigkeitsproblem und schwachen Umfragewerten zu kämpfen, da zentrale Wahlkampfversprechen wie höhere Sozialleistungen, die Rücknahme der Frührentenreform und der Reform des Arbeitslosengeldes sowie die Einführung einer Mautzone in Kopenhagen nicht erfüllt wurden. ${ }^{3}$ Dies geschah zum Teil auf Druck der Sozialliberalen, die an den Reformen der vorherigen bürgerlichen Regierung festhalten wollten und dies zur Bedingung für eine Koalition mit Sozialdemokraten und SF machten. ${ }^{4}$ Darüber hinaus hatte sich innerhalb der Sozialdemokraten der „Reformflügel“ um Helle Thorning-Schmidt, den späteren Fraktionsvorsitzenden und Industrieminister Henrik Sass Larsen sowie Finanzminister Bjarne Corydon durchgesetzt, die eine Politik „,des Dritten Weges“ repräsentieren und nicht die im

\footnotetext{
${ }^{2}$ Vgl. Flemming Juul Christiansen, Politiske Forlig i Folketinget: Partikonkurrence og Samarbejde. Aarhus 2008, S. 90.

${ }^{3}$ Vgl. Christine Cordsen, Fra start med løftebrud, in: Jyllands-Posten vom 9. Oktober 2011, S. 10; Christian Jensen, Løftebrud og vælgerbedrag, in: Information vom 7. Oktober 2011, S. 24.

${ }^{4}$ Siehe hierzu Christoph Arndt / Kees van Kersbergen, Social democracy after the Third Way: restoration or renewal?, in: Policy \& Politics, 43. Jg. (2015), H. 2, S. 203 - 220; Christoph Arndt, Die Folketingswahl in Dänemark, a.a.O. (Fn. 1), S. 151.
} 
gemeinsamen Wahlprogramm von S und SF vertretene expansive Sozialpolitik. ${ }^{5}$ Bei der Verteilung der Ministerien hatten somit die Reformer Margrethe Vestager (RV) und Bjarne Corydon (S) die Schlüsselministerien Wirtschaft und Finanzen bekommen. Die SF hatte hingegen in ihrer ersten Regierungsbeteiligung überhaupt erhebliche strategische Fehler gemacht, indem beispielsweise ihr Vorsitzender Villy Søvndal das Außenministerium wählte und daher aufgrund ständiger Abwesenheit nicht in die Tagespolitik eingreifen konnte. ${ }^{6}$ Auch aufgrund der fehlenden Erfahrung der zumeist jungen SF-Minister hatten die Sozialisten somit bereits kurz nach der Regierungsübernahme keine realistische Möglichkeit, das zusammen mit den Sozialdemokraten vor der Wahl 2011 ausgearbeitete Sozialprogramm Fair Løsning (Faire Lösung) umzusetzen.

Stattdessen wurde ein reformorientierter Kurs eingeschlagen, wo die sozialdemokratischsozialliberale Minderheitsregierung oft mit der liberalen Venstre (V), den Konservativen (K) und zuweilen auch mit der Liberale Allianz (LA) und der nationalkonservativen Dänischen Volkspartei (DF) kooperierte. Dies wurde insbesondere bei der Verabschiedung der Haushalte in 2012 und 2013 ersichtlich, wo die sozialdemokratisch-sozialliberale Regierung keine Vereinbarung mit der linksradikalen Einheitsliste aufgrund ihrer ultimativer Forderungen und Kompromissunwilligkeit einging, sondern mit Venstre und den Konservativen. ${ }^{7}$ Der Haushalt 2012 beinhaltete eine Steuerreform, welche unter anderem einen höheren Steuerfreibetrag und eine höhere Grenze für den Spitzensteuersatz vorsah, mit dem Ziel den Grenzsteuersatz zu senken und das Arbeitsangebot zu erhöhen. Die Finanzierung geschah teilweise durch Erhöhung indirekter Steuern. Entgegen der Wahlkampfversprechen kam es daher zu keiner Anpassung der Sozialleistungen an die Löhne bzw. Inflation geschweige denn zur Rücknahme der Frührentenreform oder zur Verlängerung der Bezugsdauer des Arbeitslosengeldes auf vier Jahre.

Im Gegenzug kam es zu einer weiteren Reform der Erwerbsunfähigkeitsrente (Førtidspension) um vorwiegend junge, aber eingeschränkt arbeitsfähige Versicherte zu aktivieren. Aufgrund der Zusammenarbeit der Regierung mit den bürgerlichen Parteien und des Inhaltes der Reformen kündigte die Einheitsliste darauf ihre Funktion als Tolerierungspartner (sogenannte Støtteparti)

\footnotetext{
${ }^{5}$ Vgl. Christoph Arndt / Kees van Kersbergen, Social democracy after the Third Way, a.a.O (Fn. 3), S. 211; Henrik Sass Larsen / Bjarne Corydon / Mette Frederiksen / Carsten Hansen, Socialdemokraterne er bedst for Danmark, in: Politiken vom 20. August 2012, S. 7 - 8.

${ }^{6}$ Für die parteiinterne Kritik, siehe Aage Frandsen, SF skal genfinde og genvinde sin identitet, in: Politiken vom 2. August 2012, S. 7.

${ }^{7} \mathrm{Vgl}$. Beskaftigelsesministeriet, 2012, Grundelementer i reformudspil, Kopenhagen.
} 
vorläufig auf und kündigte eine härtere Gangart gegenüber der Regierung an. ${ }^{8}$ Die klaren Brüche der Wahlkampfversprechen (Løftebrud) waren mit schlechten Meinungsumfragen für die Regierungsparteien bereits kurz nach der Regierungsübernahme verbunden, während die linke Einheitsliste und auch die DF Zugewinne durch klare Profilierung verzeichnen konnten. ${ }^{9}$

Insbesondere bei der SF war dies mit internen Unruhen verbunden, da die Partei viele Wähler an die Einheitsliste verlor und mit den historisch schlechtesten Umfragewerten konfrontiert war. ${ }^{10}$ Die interne Kritik an und Unzufriedenheit mit der zu kompromissorientierten Regierungsarbeit und dem Kurs der Partei führte schließlich zum Rücktritt des SF-Vorsitzenden Villy Søvndal am 7. September 2012 und zu einer Kampfabstimmung zwischen den Abgeordneten Annette Vilhelmsem und Astrid Krag im darauffolgenden Oktober. Vilhelmsen vertrat den traditionellen linken Flügel der Partei, war aber als Kommunalpolitikerin vorher noch nicht auf Landesebene in Erscheinung getreten, während Astrid Krag den moderaten und regierungswilligen Flügel der Partei vertrat. Mit dem Sieg Vilhelmsens waren die Konflikte jedoch nicht beendet, da Industrieminister Ole Sohn als Reaktion auf den möglichen Sieg Vilhelmsens zurücktrat und später die Partei in Richtung Sozialdemokraten verlies.

Während die SF mit parteiinternen Querelen im Herbst 2012 kämpfte, hatte es am 7-8. August 2012 einen überraschenden, aber gut vorbereiteten Wechsel an der Spitze der nationalkonservativen DF gegeben, als die langjährige Vorsitzende Pia Kjarsgaard den Vorsitz an ihren Stellvertreter Kristian Thulesen Dahl übergab. ${ }^{11}$ Der Wechsel war im Gegensatz zu den Problemen der SF nicht von Auseinandersetzungen begleitet und der rhetorisch gemäßigtere Kurs unter Kristian Thulesen Dahl bescherte der DF stetig steigende Umfragen bis zum Sommer 2014, wo die Partei relativ konstante Umfragen um die 20 Prozent konsolidierte. ${ }^{12}$

Das folgende Jahr 2013 war durch eine weitere Sozialstaatsreform geprägt, da die ThorningSchmidt-Regierung eine parteiübergreifende Übereinkunft $\mathrm{zu}$ einer grundlegenden Reform der

\footnotetext{
${ }^{8}$ Peter Burh $\phi i$ / Annette Bonde, Ministre vil tvinge unge ledige i uddannelse, in Berlingske Tidende vom, 23 Februar 2013; Marchen Neel Gjertsen, Enhedslisten: Vi er ikke længere støtteparti, in: Jyllands-Posten vom 23. Juni 2012, S. 6.

${ }^{9}$ Vgl. Sфren Risbjerg Thomsen, Historisk ringe tilslutning til S, in: Altinget vom 1. April 2012.

${ }^{10}$ Mick Kristensen, Historisk lav opbakning til SF, in: Jyllands-Posten vom 27. Juni 2012, http://jyllandsposten.dk/politik/article4736448.ece (Abruf am 7. April 2016).

${ }^{11}$ Vgl. Thomas Larsen, Kjærsgaards suveræne afsked, in: Berlingske Tidende vom 08. August 2012, S. 6; Mette Østergaard, Pia Kjærsgaard stopper som formand for DF, in: Politiken vom 7. August 2012, http://politiken.dk/indland/politik/ECE1713573/pia-kjaersgaard-stopper-som-formand-for-df/ (Abruf am 7. April 2016). ${ }_{12}$ Zur langfristigen Entwicklung der Umfragedaten siehe Berlingske Barometer - partiernes historik, http://www.politiko.dk/partiernes-historik (Abruf am 7. April 2016).
} 
Sozialhilfe (Kontanthjælp) initiierte. ${ }^{13}$ Lediglich die Einheitsliste stimmte gegen das Reformpaket, welches unter anderem die Sozialhilfe für unter 30-jährige ohne Schulabschluss oder Berufsausbildung abschaffte und durch die Ausbildungshilfe (Uddannelseshjælp) auf weitaus niedrigerem Niveau ersetzte. Darüber hinaus wurden diverse Bedingungen für den Bezug von Sozialhilfe verschärft.

Im weiteren Verlauf der Legislaturperiode $\mathrm{kam}$ es $\mathrm{zu}$ weiteren innerparteilichen Auseinandersetzungen und Skandalen, die im Austritt der SF aus der Regierung am 30. Januar 2014 kulminierten. Im August 2013 gab zunächst eine große Kabinettsumbildung von der sechs Ministerien betroffen waren. ${ }^{14}$ Am 21. November trat dann Entwicklungshilfeminister Christian Friis Bach (RV) zurück, nachdem er Unklarheiten im Zuge der sogenannten Global Green Growth Institute Affäre nicht rechtzeitig an das Folketing weitergeleitet hatte. ${ }^{15} \mathrm{Im}$ Dezember 2013 kündigte Außenminister Søvndal (SF) aus gesundheitlichen Gründen das Ende seiner politischen Karriere an, während Justizmininister B $\phi d s k o v$ (S) seinen Rücktritt einreichte, nachdem er zuvor bewusst die Unwahrheit gegenüber dem Folketing gesagt hatte. ${ }^{16}$ Die immer deutlicher werdenden Auflösungserscheinungen innerhalb der SF führten dann am 30. Januar 2014 zum Ausscheiden der Sozialisten aus der Regierung, nachdem abermals starke interne Auseinandersetzungen im Zuge des geplanten Verkaufs des Energieversorgers DONG an die amerikanische Investmentbank Goldman Sachs öffentlich sichtbar wurden. Als Konsequenz verließen weitere moderate Abgeordnete (darunter auch Krag) die SF und schlossen sich den Sozialdemokraten und der Radikalen Venstre an. Zudem trat die Vorsitzende Annette Vilhelmsen zurück und wurde durch Pia Olsen Dyhr ersetzt. $^{17}$

Die durch das Ausscheiden der SF abermals notwendige Regierungsumbildung am 3. Februar 2014 führte jedoch nicht $\mathrm{zu}$ einem Kurswechsel der neuen S-RV-Regierung, welche ein neues

\footnotetext{
${ }^{13}$ Beskaftigelsesministeriet, Aftale mellem Regeringen (Socialdemokraterne, Radikale Venstre og Socialistisk Folkeparti) og Venstre, Dansk Folkeparti, Det Konservative Folkeparti og Liberal Alliance. Aftale om en reform af kontanthjælpssystemet - flere i uddannelse og job, Kopenhagen 2013.

${ }^{14}$ Kenneth Lund / Jesper Vangkilde / Kristian Klarskov, Her er Thornings nye ministerhold, in: Politiken vom 9. August 2013, http://politiken.dk/indland/politik/ECE2042927/her-er-thornings-nye-ministerhold/ (Abruf am 6. April 2016).

${ }^{15}$ Kristeligt Dagblad, Udviklingsminister Friis Bach går af, http://www.kristeligt-dagblad.dk/danmark/medierudviklingsminister-friis-bach-g\%C3\%A5r-af (Abruf am 7. April 2016).

${ }^{16}$ Für eine Übersicht über alle acht Kabinettsumbildungen vgl. Anne Justesen / Christian Juhl Mølgaard, Her er Thornings mange rokader, in: Altinget vom 10. Oktober 2014, http://www.altinget.dk/artikel/her-er-thorningsmange-rokader (Abruf am 6. April 2016)

${ }^{17} \mathrm{Vgl.} \mathrm{Kim} \mathrm{Kristensen,} \mathrm{Et} \mathrm{kompromis} \mathrm{for} \mathrm{meget,} \mathrm{in:} \mathrm{Information} \mathrm{vom} \mathrm{31.} \mathrm{Januar} \mathrm{2014,}$ https://www.information.dk/indland/2014/01/kompromis (Abruf am 5. Februar 2015).
} 
Wachstumspaket ankündigte. ${ }^{18}$ Insgesamt war Ministerpräsidentin Helle Thorning-Schmidt eine der wenigen Konstanten in der Legislaturperiode 2011-2015, da von den im Oktober 2011 ernannten 23 Kabinettsmitgliedern lediglich vier in ihren Ämtern verblieben (neben Thorning-Schmidt waren dies Finanzminister Bjarne Corydon, Bildungsministerin Christine Antorini und Bauminister Carsten Hansen) und Thorning-Schmidt insgesamt acht Kabinettsumbildungen vollzog.

Anfang des Jahres 2014 wurde die politische Tagesordnung zunächst von der Debatte um Sozialleistungen für EU-Bürger geprägt. Kritikpunkt seitens der DF und später von Liberalen und Sozialdemokraten war, dass EU-Ausländer Anrecht auf Sozialleistungen wie etwa Kindergeld hätten, ohne dass sie jemals Steuern bezahlt haben. ${ }^{19}$

Im Mai und Juni 2014 wurde schließlich Venstre von einem Spesenskandal um ihren Vorsitzenden Lars Løkke Rasmussen erschüttert. Dieser hatte aufgrund persönlicher Finanzprobleme Kleidung und weitere Vergünstigungen von seiner Partei gestellt und erstattet bekommen, was zu andauernden parteiinternen Auseinandersetzungen, Austritten und Rücktrittsforderungen an Rasmussen führte. ${ }^{20}$ Der Spesenskandal trug dann auch zum unerwartet schlechten Wahlergebnis der Venstre bei der Europawahl vom 25. Mai 2014 bei, wo die DF mit 26,6 Prozent die stärkste Partei wurde und ihr Spitzenkandidat Morten Messerschmidt die höchste Anzahl von Personenstimmen in der Wahlgeschichte Dänemarks erhielt. ${ }^{21}$ Obwohl das schlechte Abschneiden der Liberalen Lars Løkke Rasmussen angelastet wurde, überstand dieser einen parteiinternen Machtkampf auf einer außerordentlichen Parteivorstandssitzung am 3. Juni 2014 und konnte sowohl alle Rücktrittsforderungen als auch eine eventuelle Kampfabstimmung mit seinem Stellvertreter Kristian Jensen abwehren. Auch wenn die vier bürgerlichen Parteien ihren Vorsprung in den meisten Umfragen bis zur Wahl verteidigen konnten, erholten sich Venstre und Lars Løkke Rasmussen nicht mehr von dem Popularitätsverlust im Zuge des Spesenskandals.

\footnotetext{
${ }^{18}$ Poul Tvilum, Vestager: Retningen er den samme, in: Jyllands-Posten vom 30. Januar 2014, http://jyllandsposten.dk/politik/article6447954.ece (Abruf am 5. Februar 2014); zum Wachstumspaket vgl. Finansministeriet, Danmark helt ud af krisen - virksomheder i vækst, Kopenhagen 2014.

${ }^{19}$ Kristian Thulesen Dahl, EU truer velfærden - Danske politikere gør intet, http://www.danskfolkeparti.dk/EU truer_velf\%C3\%A6rden (Abruf am. 7. April 2016).

${ }^{20}$ Vgl. Mette Østergaard, Ond stemning i Venstre, in: Politiken vom 18. Mai 2014, S. 3; Ritzaus Bureau, Løkkes sager om pengeforbrug og bilag, 20. Mai 2014, Kopenhagen, http://politiken.dk/indland/politik/politikfakta/ECE2292001/fakta-her-er-loekkes-tre-sager-om-pengeforbrug-ogbilag/ (Abruf am 7. April); Thomas Vibjerg / Christine Cordsen / Nikolaj Rytgaard, 10 minutter, der reddede Lars Løkke, in: Jyllands-Posten, S. $6-7$.

${ }^{21}$ Danmarks Statistik, Europa-Parlamentsvalg 25. Maj, https://www.dst.dk/valg/Valg1475795/valgopg/valgopgHL.htm (Abruf am 5. Februar 2016).
} 
Am 6. August 2014 gab der Vorsitzende der Konservativen Lars Barfoed seinen Rücktritt bekannt, neuer Vorsitzender wurde überraschend der Bürgermeister Viborgs Søren Pape Poulsen, welcher bisher in der Landespolitik nicht aufgetreten war und auch kein Mandat im Folketing besaß. Den Bedeutungsverlust der Konservativen seit der Folketingswahl 2011 konnte Pape Poulsen aber auch nicht aufhalten. Der Herbst 2014 läutete dann auch die Vorbereitungen auf die anstehende Wahl im nächsten Jahr ein, da Ministerpräsidentin Thorning-Schmidt im Hinblick auf den sich bereits abzeichnenden Zustrom von Asylbewerbern eine weitere Kabinettsumbildung vornahm. Arbeitsministerin Mette Frederiksen (S) wurde mit dem Justizministerium beauftragt, was politische Kommentatoren als Versuch ansahen, der sich abzeichnenden Politisierung der Asylfrage durch die DF und Venstre mit dem populärsten Kabinettsmitglied etwas entgegenzusetzen. ${ }^{22}$ Zum Jahreswechsel 2014/2015 wurde die politische Tagesordnung zunehmend von der aufkommenden Flüchtlingskrise dominiert, was die guten Umfragewerte der DF weiter bestätigte und den Wahlkampf einläutete, da politische Beobachter und die Oppositionsparteien die Ausschreibung einer Neuwahl für Mitte 2015 erwarteten. ${ }^{23}$

\section{Wahlkampf und Themen}

Der eigentliche Wahlkampf begann am 27. Mai 2015, nachdem Thorning-Schmidt Wahlen zum 18. Juni ausschrieb und der späteste mögliche Wahltermin im September lag. Die Regierungskoalition aus Sozialdemokraten und Sozialliberalen (RV) setzte auf eine zielgerichtete Expansion des Sozialstaates am Start des Wahlkampfes, war jedoch zunehmend gezwungen auf die ansteigenden Flüchtlingszahlen zu reagieren. ${ }^{24}$ Die DF machte die Asylkrise und Einwanderung zum Hauptthema in ihrem Wahlkampf, trat aber im Gegensatz zu vorherigen Wahlkämpfen jedoch relativ zurückhaltend auf. Die Sozialdemokraten und die Venstre adaptierten zunehmend die restriktiven Haltungen der DF, auch als Reaktion auf das Attentat auf ein Kulturzentrum und eine Synagoge durch einen palästinensischen Jugendlichen in Kopenhagen. ${ }^{25}$ Für die rechtsliberale Venstre war das

\footnotetext{
${ }^{22}$ Siehe Sфren Munch, Mette Frederiksen bliver ny justitsminister, in: Jyllands-Posten vom 10. Oktober 2014; Christine Cordsen, Analyse: Thorning må genstarte valgkampen, in: Jyllands-Posten vom 11. Oktober 2014, S. 1.

${ }^{23} \mathrm{Vgl}$. Niels Nфrgaard / Annette Bredmose, Thorning og Løkke skyder valgkamp i gang, Ritzaus Bureau vom 6. Januar 2015.

${ }^{24}$ Naja Dandanell, Valgkampen indtager folkemødet, in: Jyllands-Posten vom 12. Juni 2015, http://jyllandsposten.dk/politik/ECE7789927/Valgkampen-indtager-folkemødet (Abruf am. 7. April 2016).

${ }^{25}$ Jyllands-Posten, DF kræver langt færre flygtninge til Danmark 12. Juni 2015, http://jyllandsposten.dk/politik/ECE7789688/DF-kr\%C3\%A6ver-langt-f\%C3\%A6rre-flygtninge-til-Danmark/ (Abruf am 7. April 2016); Marchen Neel Gjertsen / Martin Kaae, Løkke i asyl-offensiv: "Tilstrømningen er ude af kontrol”, in: JyllandsPosten vom 9. Juni 2015, S. 4 - 5.
} 
Hauptthema neben einer restriktiveren Asylpolitik, dass es zu keiner Vergrößerung oder Wachstum des öffentlichen Sektors unter einer kommenden bürgerlichen Regierung kommen sollte, während die Liberale Allianz direkte Kürzungen im öffentlichen Dienst sowie eine weitgehende Steuerreform verlangte, was auf Widerstand seitens der DF traf.

Die SF und Einheitsliste konzentrierten ihren Wahlkampf auf die Kritik an den von der Regierung Thorning-Schmidt durchgeführten Sozialreformen und Kürzungen, was aber im Falle der SF mit einem Glaubwürdigkeitsproblem verbunden war. Ein weiterer Konkurrent im linken Lager war die vom ehemaligen sozialliberalen Kulturminister Uffe Elbak gegründete neue Partei „die Alternative“. Sie war die große und zunächst nicht ernst genommene Unbekannte im Wahlkampf 2015. Die Alternative hatte erst im März 2015 die für eine Kandidatur notwendigen 20.000 Unterschriften vorweisen können. Die Alternative gab sich ein grünes Profil und attackierte die etablierten Parteien für ihre zu stark an Sachzwängen orientierte Politik, war jedoch ansonsten schwer zu verorten, da die Partei ihre Politik in sogenannten politischen Laboratorien zusammen mit interessierten Bürgern entwickeln wollte. ${ }^{26}$ Andere Parteien und politische Beobachter kritisierten die Alternative aufgrund ihrer unrealistischen und nicht finanzierbaren Wahlversprechen wie die Einführung einer 30-Stunden Woche oder eines bedingungslosen Grundeinkommens. Anfangs wurde ein Überschreiten der Zweitprozenthürde und damit der Einzug der Alternative in das Folketing nicht als wahrscheinlich angesehen, es gab jedoch im linken Lager Befürchtungen, dass die Alternative entscheidende Stimmen für eine Wiederwahl Helle Thorning-Schmidt kosten würde, da sie vorwiegend unzufriedene RV und SF-Wähler ansprach. ${ }^{27}$

\section{Das Wahlergebnis und Wählerwanderungen}

Die Wahlen vom 18. Juni 2015 brachten einen hauchdünnen Regierungswechsel mit sich, da die vier bürgerlichen Parteien zusammen die entscheidenden 90 Mandate bekamen, waren aber zugleich ein politischer Erdrutsch (vgl. Tabelle 1). Die Nettovolatilität im Sinne Mogens N. Pedersens, d.h. die Nettowählerwanderungen zwischen den konkurrierenden Parteien, erreichte den

\footnotetext{
${ }^{26} \mathrm{Vgl}$. Morten Skarbaek, Jeg siger dig, det kommer til at blive ret interessant, in: Politiken vom 13..März 2015, http://politiken.dk/indland/politik/ECE2585777/jeg-siger-dig-det-kommer-til-at-blive-ret-interessant/ (Abruf am 5. Februar 2016).

${ }^{27} \mathrm{Vgl}$. Ingelise Skrydstrup, Nu er det alvor med Alternativet - kan blive Thornings knald eller fald, in: BT vom 25. März 2015, http://www.bt.dk/politik/nu-er-det-alvor-med-alternativet-kan-blive-thornings-knald-eller-fald (Abruf am. 5. Februar 2016).
} 
höchsten Wert seit der Erdrutschwahl von $1973 .^{28}$ Die rechtsliberale Venstre verlor mehr als sieben Prozentpunkte, fuhr ihr schlechtestes Resultat seit 25 Jahren ein und war erstmals seit 15 Jahren nicht mehr die stärkste Partei im Folketing. Die Sozialdemokraten als nun stärkste Partei konnten etwas überraschend leichte Zugewinne verzeichnen und hatten somit die Stimmverluste aus den Umfragen seit 2011 mehr als kompensiert, was jedoch hauptsächlich auf Kosten ihrer (ehemaligen) Koalitionspartner SF und RV geschah, welche beide etwa 5 Prozentpunkte einbüßten und sich mehr als halbierten. Die DF verzeichnete mit 21,1 Prozent der Stimmen das beste Wahlresultat ihrer Geschichte und wurde zur größten bürgerlichen Partei, was hauptsächlich durch eine Wählerwanderung von unzufriedenen Venstre-Wählern innerhalb des bürgerlichen Blocks zurückzuführen ist (vgl. Tabelle 2). Hier waren neben dem Thema Einwanderung die niedrigen Popularitätswerte Løkke Rasmussens ein entscheidendes Wanderungsmotiv. ${ }^{29}$

${ }^{28}$ Zur Definition der Nettovolatilität, vgl. Mogens N. Pedersen, The Dynamics of European Party Systems: Changing Patterns of Electoral Volatility, in: European Journal of Political Research, 7. Jg. (1979), H.1, S. 1 - 26; für die Resultate, Kasper Møller-Hansen / Rune Stubager Folketingsvalget 2015, in: dies. (Hrsg.), Oprør fra udkanten Folketingsvalget 2015. Im Druck, Kopenhagen 2017.

${ }^{29} \mathrm{Vgl}$. Kasper B. Andreasen / Said Nuh, Så mange vælgere har Thulesen Dahl 'stjålet' fra Løkke, TV2/Nyheder, http://nyheder.tv2.dk/valg2015/2015-06-19-grafik-saa-mange-vaelgere-har-thulesen-dahl-stjaalet-fra-loekke (Abruf am. 20. Juni 2015). 


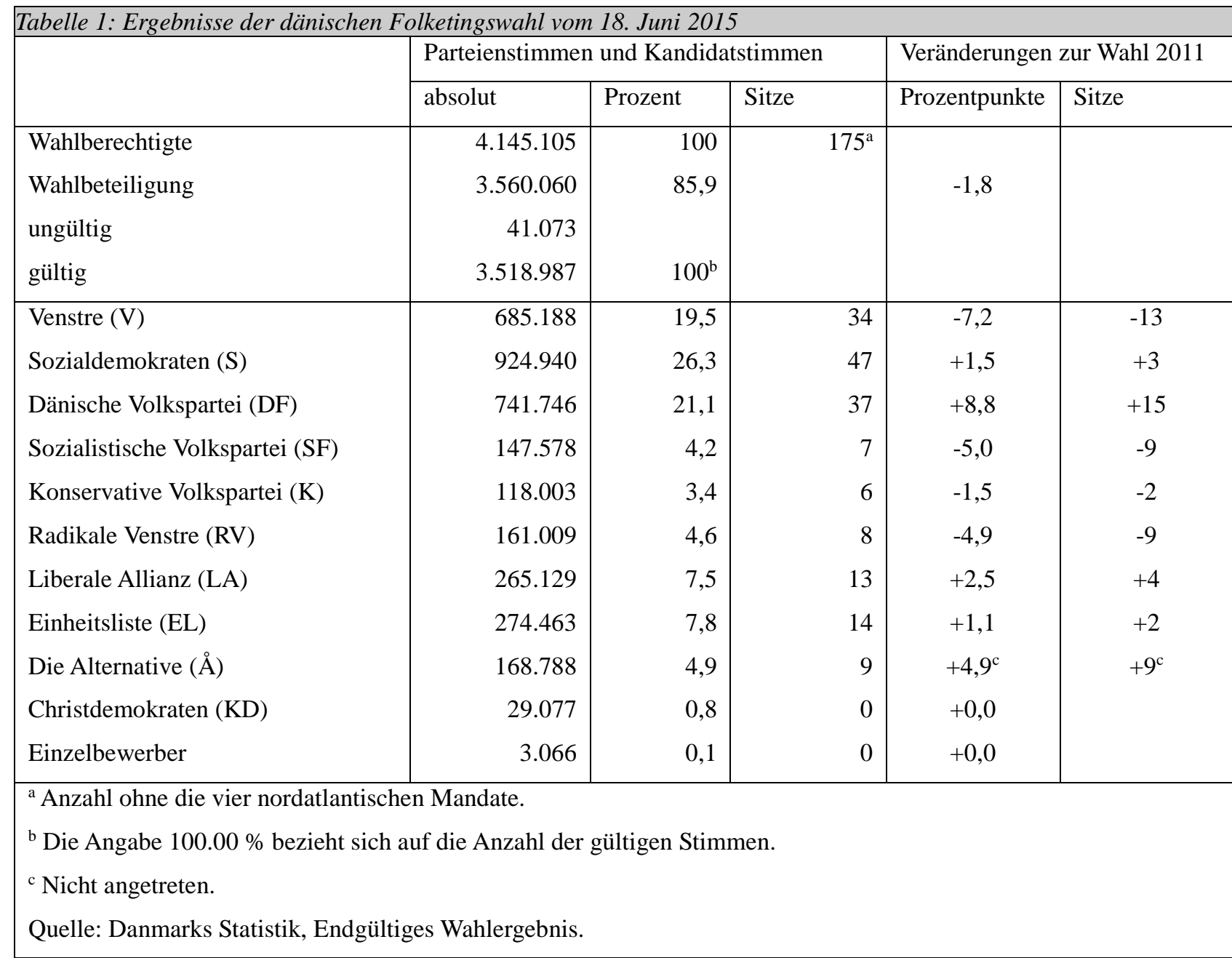

Im Gegensatz zu den Wahlen 1998 und 2001 lösten die Sozialreformen der sozialdemokratischsozialliberalen Regierung nur geringe Wählerwanderungen zwischen den Blöcken aus, nachdem vergleichbare Reformen unter dem Sozialdemokraten Poul Nyrup Rasmussen für die Abwanderung von sozialdemokratischen Stammwählern zu V und DF verantwortlich waren. ${ }^{30}$

Die Alternative überwand überraschend deutlich die Zweiprozenthürde und gewann mit fast fünf Prozent der Stimmen vorwiegend unzufriedene linke und sozialliberale Wähler sowie Wähler der Einheitsliste (vgl. Tabelle 2). Die abermaligen Verluste der Konservativen und das schwache Abschneiden der Sozialliberalen markierten somit auch das schlechteste Resultat für die vier alten

\footnotetext{
${ }^{30}$ Zur Bedeutung der Sozialreformen für die Wählerwanderungen bei der Wahl 2015 Christoph Arndt / Carsten Jensen, Partivalg og holdninger til velfærdsstaten, in: Kasper Møller-Hansen / Rune Stubager (Hrsg.), Oprør fra udkanten Folketingsvalget 2015. Im Druck, Kopenhagen 2017; für die vorherigen Wählerwanderungen Christoph Arndt, Denmark, in: ders., The Electoral Consequences of Third Way Reforms: Social Democracy's Transformation and Its Political Costs, Amsterdam 2013.
} 
Parteien (S, V, K und RV) sowie die größte elektorale Nettoverschiebung seit den Erdrutschwahlen 1973. Analog zur Fortschrittspartei 1973 wurde ihr Nachfolger, die DF, die zweitgrößte Partei und hatte entscheidenden Einfluss auf die kommende Regierungsbildung im bürgerlichen Lager. Der Vorsitzende der DF, Kristian Thulesen Dahl, bekam mit 57.371 persönlichen Stimmen auch die meisten persönlichen Stimmen aller Kandidaten und übertraf somit Ministerpräsidentin Helle Thorning-Schmidt (42.412 persönliche Stimmen), welche auch nur knapp vor der Spitzenkandidatin der Einheitsliste, Johanne Schmidt-Nielsen (40.425), landete. Der kommende Ministerpräsident der Venstre, Lars Løkke Rasmussen, landete mit 33.393 auf dem vierten Platz, nachdem er 2011 noch 56.285 persönliche Stimmen verzeichnen konnte. ${ }^{31}$

\begin{tabular}{|c|c|c|c|c|c|c|c|c|c|c|c|}
\hline & & \multicolumn{10}{|c|}{ Wahl 2015} \\
\hline & & V & $\mathrm{S}$ & $\mathrm{DF}$ & SF & $\mathrm{K}$ & $\mathrm{RV}$ & LA & EL & $\mathrm{A}^{\mathrm{b}}$ & $\begin{array}{l}\text { Nicht- } \\
\text { wähler }\end{array}$ \\
\hline \multirow{10}{*}{$\underset{\substack{n\\
}}{\stackrel{n}{\pi}}$} & \multirow{10}{*}{$\begin{array}{l}\text { V } \\
\text { S } \\
\text { DF } \\
\text { SF } \\
\text { K } \\
\text { RV } \\
\text { LA } \\
\text { EL } \\
\text { Nichtwähler } \\
\text { Erstwähler }\end{array}$} & 58,8 & 6,3 & 15,4 & 0,6 & 2,5 & 1,1 & 12,0 & 0,3 & 1,6 & 1,3 \\
\hline & & 3,1 & 73,5 & 4,5 & 4,5 & 0,3 & 2,0 & 1,4 & 3,7 & 4,4 & 2,7 \\
\hline & & 6,5 & 4,7 & 82,2 & 0,7 & 0,0 & 0,0 & 2,8 & 0,7 & 0,7 & 1,7 \\
\hline & & 0,0 & 29,1 & 4,6 & 29,3 & 1,0 & 0,4 & 0,4 & 22,1 & 10,9 & 2,2 \\
\hline & & 13,9 & 9,5 & 12,6 & 1,0 & 40,2 & 3,1 & 13,4 & 3,8 & 0,0 & 2,6 \\
\hline & & 7,0 & 23,5 & 4,5 & 2,8 & 3,0 & 35,7 & 4,3 & 3,3 & 14,8 & 1,2 \\
\hline & & 15,5 & 3,5 & 9,8 & 0,0 & 5,4 & 1,6 & 61,5 & 1,8 & 0,0 & 0,9 \\
\hline & & 1,7 & 10,4 & 4,0 & 4,5 & 0,0 & 1,2 & 0,6 & 57,3 & 16,9 & 3,4 \\
\hline & & 2,9 & 11,9 & 9,8 & 3,3 & 4,1 & 3,3 & 9,5 & 7,1 & 2,8 & 45,4 \\
\hline & & 16,6 & 14,2 & 12,1 & 7,6 & 1,0 & 8,5 & 16,5 & 9,8 & 6,2 & 8,5 \\
\hline \multicolumn{12}{|c|}{$\begin{array}{l}\text { a Prozentpunkte beziehen sich auf Wahlentscheidung in } 2015 \text { aufgeschlüsselt nach Wahlentscheidung von } 2011 . \\
\text { b } 2011 \text { nicht angetreten. } \\
\text { c Christdemokraten (KD) nicht aufgeführt aufgrund zu geringer Fallzahl } \\
\text { Quelle: Eigene Berechnungen basierend auf dänischer Wahlstudie } 2015 .\end{array}$} \\
\hline
\end{tabular}

\section{Regierungs- und Oppositionsbildung sowie Aussicht}

Die hauchdünne bürgerliche Mehrheit vom 18. Juni 2015 brachte in der Folge kurze aber schwierige Koalitions- und Tolerierungsverhandlungen mit sich. Ein Grund war, dass die DF stärker als die „,natürliche“ Regierungspartei Venstre geworden war. Somit würde in einer etwaigen Koalition von Venstre und DF der größere Regierungspartner zum einen nicht den Ministerpräsidenten stellen und zum anderen keinerlei Regierungserfahrung aufweisen. Der Vorsitzende der DF, Kristian Thulesen Dahl, hatte darüber hinaus keine eindeutige Aussage zu einer möglichen Regierungsbeteiligung der DF vor der Wahl gemacht und nach der Wahl

\footnotetext{
${ }^{31}$ Vgl. Danmarks Statistik, Folketingsvalg Torsdag 18. Juni 2015, Valgte Kandidater Og Stedfortrædere, https://www.dst.dk/valg/Valg1487635/kandstat/kandstat.htm (Abruf am 11. April 2016).
} 
weitgehende Forderungen für eine Koalitionsbeteiligung der DF gestellt. Eine maßgebliche strategische Überlegung innerhalb der DF war hierbei, dass sie als große Støtteparti mehr Einfluss außerhalb der Regierung erhalten könnte als als Regierungspartei, die zu viele Kompromisse eingehen müsste, während wie im Falle der SF in der vorangegangenen Legislaturperiode niemand Ministererfahrung besaß. ${ }^{32}$ So gab es schließlich vier Regierungskonstellationen, die vor der Sondierungsrunde mit Königin Margrethe II als realistisch angesehen wurden: Eine mitte-rechts Mehrheitskoalition aus allen vier bürgerlichen Parteien (V, DF, LA, K), eine Venstre-DFMinderheitsregierung, eine liberale Minderheitsregierung aus Venstre und Liberaler Allianz und eine reine Venstre-Minderheitsregierung, die von allen anderen bürgerlichen Parteien toleriert wird. ${ }^{33}$ Nachdem alle Versuche zur Bildung einer Koalitionsregierung an den weitgehenden Forderungen der DF im Bereich der Einwanderungs- und EU-Politik und der Liberalen Allianz im Bereich der Steuerpolitik gescheitert waren, wurde Lars Løkke Rasmussen (V) am 28. Juni schließlich von Königin Margrethe II mit der Bildung einer reinen Venstre-Regierung beauftragt. ${ }^{34}$ Diese Regierung verfügt lediglich über 34 der insgesamt 179 Mandate und erinnert an die VenstreMinderheitsregierung Poul Hartlings, die nach den Erdrutschwahlen 1973 gebildet wurde. Løkke Rasmussens Minderheitskabinett benötigt stets alle 90 Mandate der bürgerlichen Parteien, wenn keine lagerübergreifenden parlamentarischen Mehrheiten gebildet werden können, was den Einfluss von DF und Liberaler Allianz stärkt und den schwachen Konservativen Möglichkeiten zur Profilierung gibt.

Das Kabinett Løkke Rasmussen III umfasst nur noch 16 Ministerien, nachdem die vorherige mittelinks Regierung noch 22 Ministerien umfasste. Die Verkleinerung des Kabinettes ist der geringen Anzahl der Venstre-Mandate geschuldet und wurde durch Zusammenlegung diverser Aufgabenbereiche erreicht.

\footnotetext{
${ }^{32}$ Ritzaus Bureau, DF føler sig ikke tvunget til at gå i regering, Kopenhagen 19. Juni 2015; Sine Riis Lund, DF: Derfor gik vi ikke i regering, in: Altinget vom 5. August 2015, http://www.altinget.dk/artikel/df-derfor-gik-vi-ikke-i-regering (Abruf am 11. April 2016).

${ }^{33}$ Vgl. Nilas Heinskou / Morten Skarbak / Jesper Thobo-Carlsen, Otte mulige og et par realistiske regeringsscenarier, in: Politiken vom 21. Juni 2015, S. 3.

${ }^{34}$ Jakob Stig Jørgensen / Frederik Buhl Kristensen, Løkke opgiver at få andre med: Danner ren V-regering, in: Politiken vom 26. Juni 2015, http://politiken.dk/indland/politik/folketingsvalg2015/ECE2733511/loekke-opgiver-at-faa-andremed-danner-ren-v-regering (Abruf am 26. Juni 2015); Steen A. Jørgenssen, Løkke vil danne ren Venstre-regering, in: Jyllands-Posten vom 26. Juni 2015, http://jyllands-posten.dk/politik/ECE7832923/L\%C3\%B8kke-vil-danne-renVenstre-regering (Abruf am 26. Juni 2015).
} 


\begin{tabular}{|c|c|}
\hline \multicolumn{2}{|c|}{ Tabelle 3: Die dänische Regierung nach der Folketingswahl 2015} \\
\hline Ministerpräsident & Lars L $\phi k k e$ Rasmussen (V) \\
\hline Wirtschaft & Troels Lund Poulsen (V) \\
\hline Äußeres & Kristian Jensen $(\mathrm{V})$ \\
\hline Finanzen & Claus Hjort Frederiksen (V) \\
\hline Justiz & Søren Pind (V) \\
\hline Soziales und Inneres & Karen Ellemann $(\mathrm{V})$ \\
\hline Steuern & Karsten Lauritzen $(\mathrm{V})$ \\
\hline Transport und Bau & Hans Christian Schmidt $(\mathrm{V})$ \\
\hline Forschung & $\begin{array}{l}\text { Esben Lunde Larsen (bis 29. Februar 2016, V) } \\
\text { Ulla Tornces (ab 29. Februar 2016, V) }\end{array}$ \\
\hline Arbeit & Jфrn Neergaard Larsen $(\mathrm{V})$ \\
\hline Kinder, Bildung und Gleichstellung & Ellen Trane Nфrby $(\mathrm{V})$ \\
\hline Ausländer, Integration und Wohnungsbau & Inger Stфjberg (V) \\
\hline Energie, Versorgung und Klima & Lars Christian Lilleholt $(\mathrm{V})$ \\
\hline Gesundheit und Ältere & Sophie L Lhde (V) \\
\hline Verteidigung und nordische Zusammenarbeit & $\begin{array}{l}\text { Peter Christensen (V) (bis 29. September Carl } \\
\text { Holst, V) }\end{array}$ \\
\hline Landwirtschaft, Umwelt und Ernährung & $\begin{array}{l}\text { Eva Kjer Hansen (bis 27. Februar 2016, V) } \\
\text { Esben Lunde Larsen (ab 29. Februar 2016, V) }\end{array}$ \\
\hline Kultur und Kirche & Bertel Haarder $(\mathrm{V})$ \\
\hline
\end{tabular}

Bereits kurz nach dem Amtsantritt trat der Minister für Verteidigung und nordische Zusammenarbeit, Carl Holst, zurück, nachdem er unerlaubterweise Beamte aus der Region Süddänemark, wo er zuvor als Regionsvorsteher amtierte, für die Wahlkampfunterstützung einsetzte. Einen weiteren Rückschlag erlitt die neue Venstre-Regierung bei der Volksabstimmung über die Rechtsvorbehalte am 3. Dezember 2015. Dänemark verfügte seit dem EdinburghAbkommen von 1993 über eine Ausnahmeregelung bezüglich der Teilnahme an der gemeinsamen europäischen Sicherheits- und Justizpolitik, über die bei einer Volksabstimmung am 3. Dezember neu abgestimmt werden sollte. ${ }^{35}$ Die Venstre-Regierung unterstützte zusammen mit Sozialdemokraten, Sozialliberalen, Konservativen und der Alternative eine Abschaffung der Rechtsvorbehalte, da dies eine Zusammenarbeit mit europäischen Institutionen wie Europol in der Kriminalitätsbekämpfung erleichtern würde. Die DF, Liberale Allianz und die Einheitsliste empfahlen den Wählern für die Beibehaltung der dänischen Rechtsvorbehalte zu stimmen, da innere Sicherheit und Justiz als innerdänische Angelegenheiten ohne Souveränitätsabgabe an die EU verbleiben sollten. Somit waren sowohl das linke als auch das bürgerliche Lager gespalten was die Haltung zu den Rechtsvorbehalten anging. 53.1 Prozent der Wähler stimmten bei einer

\footnotetext{
${ }^{35}$ Vgl. Helmut Steuer, Dänemark und sein Referendum, in: Handelsblatt vom 3. Dezember 2015, Ja oder nein zu mehr Europa?, http://www.handelsblatt.com/politik/international/daenemark-und-sein-referendum-ja-oder-nein-zu-mehreuropa/12673242.html (Abruf am. 4. Dezember 2015).
} 
Wahlbeteiligung von 72 Prozent schließlich für die Beibehaltung der Rechtsvorbehalte, was als Niederlage für die neue Regierung angesehen wurde, welche sich stark für die Abschaffung eingesetzt hatte. ${ }^{36}$

Trotz des Fehlstartes konnte Lars Løkke Rasmussens Minderheitsregierung zuvor den Haushalt für 2016 am 19. November 2015 mit den Stimmen der DF, der Liberalen Allianz und der Konservativen durchsetzen. Der erste Haushalt der neuen Regierung wurde als „blau“ und somit bürgerlich charakterisiert, da er einige kleinere Steuersenkungen, eine Wiedereinführung der Deckelung der Sozialhilfe (Kontanthjælpsloft) und Mehrausgaben für die Polizeiarbeit und Altenpflege vorsah, was zuvor von Venstre, DF und Liberaler Allianz im Wahlkampf versprochen wurde. ${ }^{37}$

Im Frühjahr 2016 kam es aber dann zu ersten größeren Uneinigkeiten in der Steuerpolitik, als der Vorsitzende der Liberalen Allianz Anders Samuelsen das Überleben der Regierung von der Durchführung einer umfassenden Steuerreform abhängig machte. ${ }^{38}$ Kurze Zeit später verlangten die Konservativen den Rücktritt von Landwirtschaftsministerin Eva Kjer Hansen welche bei der Vorlage eines Gesetzpaketes (Landbrugspakke) unklare Angaben zum zukünftigen Stickstoffausstoß der dänischen Landwirtschaft gemacht hatte. Dies wurde von politischen Kommentatoren als verzweifelter Profilierungsversuch der Konservativen angesehen, da dem Landbrugspakke zuvor keinerlei politische Relevanz zugemessen wurde und der erzwungene Rücktritt Eva Kjer Hansens am 27. Februar 2016 das Arbeitsverhältnis zwischen Liberalen und Konservativen deutlich belasten würde. ${ }^{39}$ Im Herbst 2016 erneuerte der Vorsitzende der Liberalen Allianz Anders Samuelsen seine Forderung nach einer umfassenden Steuerreform. Falls der

\footnotetext{
${ }^{36}$ Vgl. Christine Cordsen, Vælgerlussingen er et ubehageligt hattrick til Lars Løkke Rasmussen, in: Jyllands-Posten vom 4. Dezember 2015, S. 2.

${ }^{37}$ Jyllands-Posten, Blå Finanslov, in Jyllands-Posten vom 21. November 2011, http://jyllandsposten.dk/debat/leder/ECE8233072/B1\%C3\%A5-finanslov/ (Abruf am 11. April 2016); Finansministeriet, Aftale mellem regeringen, Dansk Folkeparti, Liberal Alliance og Det Konservative Folkeparti: Finansloven for 2016, Kopenhagen 2015.

${ }^{38}$ Vgl. Jakob Hvide Beim / Morten Skarbak, Her er tallet, der truer med at sprænge blå blok, in: Politiken vom 12. Januar 2016, S. 5.

${ }^{39}$ Lars Nørgaard / Michael Lund / Chris Jensen, Hvorfor det kom dertil, at der ikke længere er tillid til Eva Kjer Hansen, er dybest set ganske uforståeligt, in: Berlingske Tidende vom 23. Februar 2016, http://www.b.dk/fraeditorial/hvorfor-det-kom-dertil-at-der-ikke-laengere-er-tillid-til-eva-kjer-hansen-er- (Abruf am 24. Februar 2016); Für eine Übersicht über den Verlauf vgl. Ritzaus Bureau, Kronologi: Eva Kjer Hansen-sagen dag for dag, 29. Februar 2016, Kopenhagen.
} 
Spitzensteuersatz nicht um mindestens fünf Prozentpunkte gesenkt würde, so würde die Liberale Allianz ihre Tolerierung der Venstre-Regierung aufkündigen und Neuwahlen riskieren. ${ }^{40}$

Bereits die ersten Monate nach dem Regierungswechsel zeigten somit die blockinternen Differenzen mit denen Ministerpräsident Rasmussen vermutlich bis zur nächsten Wahl spätestens im Juni 2019 konfrontiert sein wird, sofern er keine vorzeitigen Neuwahlen ausschreibt oder die Liberale Allianz ihre Tolerierung aufkündigt. Rasmussens liberale Partei Venstre hatte innerhalb von 18 Monaten drei wichtige Abstimmungen verloren, wurde aber dennoch einzige Regierungspartei nach dem schwachen Abschneiden bei der Folketingswahl vom 18. Juni 2015, da sich alle anderen Regierungsoptionen schnell zerschlugen. Die Konstellation mit einer Einparteienminderheitsregierung kann sich nur dann als stabil erweisen, wenn es Venstre gelingt alle drei Støtteparteien bei Laune halten zu können. Die DF ist hier mit der Herausforderung konfrontiert, eine so große und relativ heterogene Wählerkoalition anzusprechen, welche aus DFStammwählern aus der Arbeiterschaft sowie vielen vormaligen liberalen Wählern besteht, die die DF hauptsächlich aufgrund ihrer Unzufriedenheit mit dem Venstre-Vorsitzenden Lars Løkke Rasmussen gewählt haben und nicht aus Überzeugung.

Bei den Sozialdemokraten wurde nach dem Rücktritt Helle Thorning-Schmidts am Wahlabend ein deutlicher Kurswechsel bei der Asyl- und Zuwanderungspolitik eingeleitet. Die neue Parteivorsitzende Mette Frederiksen und der Fraktionsvorsitzende Henrik Sass Larsen erklärten, dass der vormals liberale Kurs der Partei in den 1990er Jahren ein Fehler war und dass die Sozialdemokraten alles dafür tun würden, die Zuwanderung nichtwestlicher Migranten zu begrenzen. $^{41}$ Eine Implikation des restriktiveren Kurs der Sozialdemokraten in der Zuwanderungsfrage ist, dass die Partei zukünftig weniger anfällig für Stimmenverluste an Venstre und DF ist, sobald Zuwanderung die politische Tagesordnung dominiert. Dies dürfte den Sozialdemokraten die Chance eröffnen, ihre derzeitige Position als stärkste Partei zu konsolidieren, während es gleichzeitig zu größeren Unstimmigkeiten innerhalb des roten Blocks kommen dürfte, da Sozialliberale und Sozialisten hier ein deutlich liberales Profil aufweisen.

\footnotetext{
${ }^{40} \mathrm{Vgl}$. Sфren Domino, 5 procentpoint lettelse af topskat er absolut smertegrænse for LA, in: Berlingske Tidende vom 12. August 2016, S. 4.

${ }^{41}$ Anders Legarth Schmidt, Mette Frederiksen lægger sig fladt ned: Jeg tog fejl om flygtninge og integration, in Politiken vom 20. Januar 2016, http://politiken.dk/udland/fokus_int/Flygtningestroem/ECE3024826/mettefrederiksen-laegger-sig-fladt-ned-jeg-tog-fejl-om-flygtninge-og-integration/ (Abruf am. 21. Januar 2016); Henrik Sass Larsen, Vi vil gøre alt for at begrænse antallet af ikkevestlige flygtninge og indvandrere, in Politiken vom 18. Dezember 2015, http://politiken.dk/debat/ECE2982956/vi-vil-goere-alt-for-at-begraense-antallet-af-ikkevestligeflygtninge-og-indvandrere/ (Abruf am 19. Dezember 2015).
} 
Eine weitere offene Frage ist, ob sich die Alternative als kurzfristiges Protestphänomen wie die deutsche Piratenpartei herausstellt oder sich als neue ökolibertäre Partei fest im Parteienspektrum Dänemarks etablieren kann, welche primär um die Wähler der RV und SF konkurriert. Letzteres würde eine Spaltung des ökologischen, gesellschaftspolitisch liberalen und großstädtischen Wählermilieus implizieren, wie sie auch bei der letzten Stortingswahl in Norwegen zu beobachten war. $^{42}$

${ }^{42}$ Zur Positionierung der Miljøparti in Norwegen Anders Ravik Jupskås, Miljøpartiet de grønne og det «politiske rommet», in: Norsk Statsvitenskapelig Tidsskrift 29. Jg. (2013), H. 2, S. 131-143. 